\title{
Temperature and Humidity Management of the Storage Houses of Food Using Data Logger
}

\author{
Abdulrahman Alkandari ${ }^{1+}$, Altaf Alshammari ${ }^{2}$ and Samer Moein ${ }^{3}$ \\ ${ }^{1}$ Computer Department, College of Basic Education, (PAAET), Kuwait \\ ${ }^{2}$ Om Mabad Middle School, Ministry of Education, Kuwait \\ ${ }^{3}$ Electrical and Computer Engineering, University of Victoria, Canada
}

\begin{abstract}
Because of commercial fraud, and high and low temperature and humidity in the environment that lead to food spoilage and this may affect the health of the consumer. People need an effective way to maintain the safety of food products and consumer health. To control this problem, this paper proposed a solution using the data logger by connecting it to a computer. Data logger is a device that records the temperature and humidity of food products at regular times (depending on the minutes, hours, date), it displays them as fees graphic schemes, therefore, and it becomes easy to read. The results of these papers showed that for each environment different temperature and humidity. The data logger has proved its effectiveness in accurately recording the readings to prove the safety of the product by taking readings regularly, and this helps in controlling the problem of damage of products.
\end{abstract}

Key words: data logger system, mobile application, UML.

\section{Introduction}

Lord Kelvin says, "When you can measure what you are speaking about, and express it in numbers, you know something about it, when you cannot express it in numbers, your knowledge is of a meagre and unsatisfactory kind; it may be the beginning of knowledge, but you have scarcely, in your thoughts advanced to the stage of science" [1]. It is known that the capacity of the human self-limited. However, increasing human capabilities led to invent a lot of scientific instruments that help to understand and examine objects and phenomena surrounding, that is the most important devices that helped the man to come to the realities of things are gauges that evolved dramatically in the context of the massive industrial development that followed the Second World War.

As we cannot separate between scientific advances and industrial progress and that because any scientific discovery followed by discoveries in the field of industry, technology and the attendant development of new ways and means to carry out the measurement or observation or recording. Thus, increased the variables that need to be a precise measurement, and increased attention to improve methods of measurement and development Instrumentation and even in the lives of their human interest shifted to the attention of the kind of type and quantity together. The meaning of quantum measurement and measurement requires the use of the device, and use knowledge to use right what is the measurement. It is find the amount of physical or variable physicist or estimate the case using a suitable or appropriate tool. And if the user device standard universally agreed considered the measurement calibration process, the quantity will be measured of the standard amount. But if the device is not a standard, it must compare the measurement process with the standard amount used in this device, which is calibrated before. And calibration devices

\footnotetext{
+ Corresponding author. Tel.: +96599868868.

E-mail address: aam_alkandary@yahoo.com.
} 
used to compare with normative universally agreed-upon terms of accuracy and reserved under specific environmental conditions. There is universal agreement on the measurement titer and the units of measurement Fahrenheit and Celsius or humidity. Any measurement process requires the use of a measure and despite the fact that there are a large number of devices, which are not electric [2]. But the general trend in the industry and research laboratories has become an attempt to use electrical appliances. Also it called electronic devices and cause this trend is the ease of use of electrical devices and convert them to digital devices and easily connect to other device help to record the readings or saved in computer peripherals, making it easier and statistical calculations.

\section{Problem Discerption}

Each adoption of maintaining good food to see the proper storage of each type of food to protect it from pollution due to high humidity and temperature are favourable for growth and reproduction of microbes and viruses, especially if the storage process is bad. Control the storage environment in terms of temperature is considered a most common ways effective to reduce the number of microbes, prevent their spread and ruin food or medical during storage. Therefore, it must provide all the nutrients in the proper temperatures, either refrigerated or frozen, and its importance to human health must be preserved and taken care of. These problems do not occur all intentionally but may have found a technical malfunction or electric in refrigerators. This paper presents an idea of a device measured the temperature, to help organizations and other that need to take caution and precaution to maintain human health. Therefore, to solve those problems and maintaining the health of these human damages, (USB Temperatures and humidity sensor or Data Logger) device has been chosen. In addition, to ensure the quality of foods as they move between states, also to achieve transparency and to ensure consumer confidence.

\section{Related Works}

There are many applications and researches regarding the measurement of temperature Measurements of skin temperatures.

Norman, R., J. Henfling, and D. Blackwell, in 1996, presented the most important study made for the use of Data Loggers sensor in measuring the temperature of the human body skin. There are two ways of the wired sensor. There are many studies in this regard. This study aims mainly at describing how I-Button is used in the shape of wireless for measuring the temperature of human skin. This article describes a wireless temperature system for human skin this study deals with proof of the Data Logger and its application on the human skin temperature [4].

In 2006, van Marken Lichtenbelt, Wouter D., et al. presented a study which has been validated by the accuracy of the Data logger sensor which can reach the amount of $0.09{ }^{\circ} \mathrm{C}\left(-0.4{ }^{\circ} \mathrm{C}\right.$ at most). Such use of the Data Logger these properties can be developed for more and better result by calibration [5].

According to the Kit L. Yam study (October 2009), the use of Data Loggers can include many complementary technology applications such as approximately. In addition, smart cards, which differ from other temperature sensors, the Data Logger, exceeded the limitation of the digital memory. Also it showed via experiments, the accuracy of using I buttons in measuring temperature via using more nearly 30 Data Loggers sensors, they were all put in the water bath, coated in a net and mesh the same was done with a calibrated thermostat. The water bath is to be contained in a constant heat level for 15 minutes. The Data Logger proved their own ability to measure temperature more than the usual thermostat. Another experiment is showing the validity of the Data Logger via having two vessels of water with foam polystyrene filled all with different temperature water. The number of 8 Data Logger sensors with calibrated thermometer which are to be put loosely in a tube holder. There was enough room and space among the Data Logger to be surrounded with water The Data Loggers are submerged into the water $=8.7^{\circ} \mathrm{C}$ and next, immediately after the prompt measurement, put in another water vessel with water $=41.3^{\circ} \mathrm{C}$. The example rate was set to one taster per minute. [6]

In 2005, JOHN PORTER, PETER ARZBERGER and others in their paper described the Data Logger sensor as one of the developed sensors, which is used automatically, and according to the net with wireless 
for different uses even in temperature controlling. For the study, a sensor of Data Logger type can be used for measuring physical aspects including temperature and other biological functions via converting the signal it traced and detected to be in the shape of information or data. Data Logger can be seen as a bridge between the physical world in the electrical and electronic world for measuring and monitoring experience [7].

\section{Data Logger}

A data logger is a device with processor-controlling storage memory unit, which receives and collect the data in a certain Sensor temperature, and humidity and stores it on a storage medium memory. This can be done by software, if the present data to be logged. For example, information within a computer already in digital form and only need to be treated. If the information is only in the non-electrical form available, the data logger from a special combined with hardware sensors can be made to capture the physical measurement data over a certain period of time, such as: temperatures, voltages, and accelerations. Some data recorders can be reached with a personal computer to capitalize on and activate the software, the device could be with (keyboard, display), and the user can use it as a standalone device as shown in Fig. 1, which shows the Data Logger system.

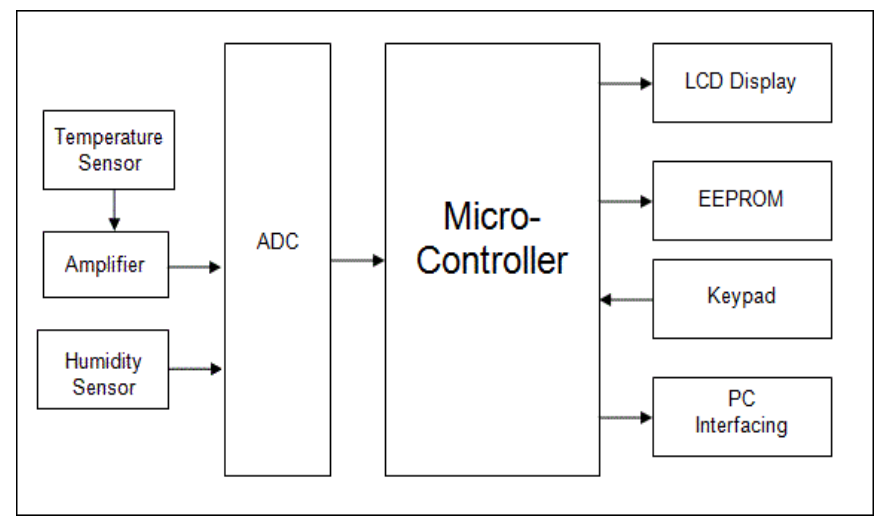

Fig. 1: Data logger system.

There are many data recorders, which requires the development of an application on your computer so that data is processed, and these records are characterized as the high-resolution, but have no attribution error is limited and can use the application comes with the device it where it is to identify the data and applied using a computer program. There are some data recorders, which contain information, analysis alone without resorting to computer. One of the main benefits of the use of data recorders is the ability to collect data on the basis of 24 hours automatically. When activated, the deployment and are usually put data recorders and left without processing to measure and record information for the duration of the monitoring period. This allows for a comprehensive and accurate pictures of the environmental conditions being monitored, such as air temperature and relative humidity. Depending on the use, governed by a quality management system sometimes need to calibrate compared to global standards and protocols official commitment to verification and validation can be based Options temperature data recorders on many factors, such as:

\section{Uses of the Data Logger}

- Environmental Monitoring: Can be taken as data recorders, independent sites varied that cannot be easily supported with temperature monitoring fixed. Applications, data, logger temperature model include the mountains and deserts, forests, mines, and flows of ice, etc. Using data recorders also portable in the industrial laboratories and unwanted conditions for registration in the independent [9].

- Using temperature data logger in factories monitoring and recording of temperature: It is vital in many manufacturing processes. In the food industry often uses temperature data logger to ensure that is stored unprocessed foods at the right temperature in cold stores. It is also used in furnaces and production lines to ensure that the food has been heated to the correct temperature the length of time required [9]. 
- Monitor shipments Temperature: There are many products in food and medical that needs to keep it safe during the process of shipping. Because of the poor storage process can happen some disrepair and ruin, or a change in the chemical structure where it should be on companies specialized freight operations to make sure to adjust the temperature and humidity of the shipping carriers. As high temperatures or moisture may cause the destruction of products is compelling put control devices and sensors to record data to ensure quality safety product upon arrival. There are studies in different indicate that the walls of the tankers non-isolated well may be affected by the rapid factors change the atmosphere and temperatures to affect negatively on the products contained inside therefore be put your data logger to monitor changes in temperature and humidity in the tanker [9].

\section{Program Work Flow}

In this section, images from the system will be displayed with a simplified explanation of each image.

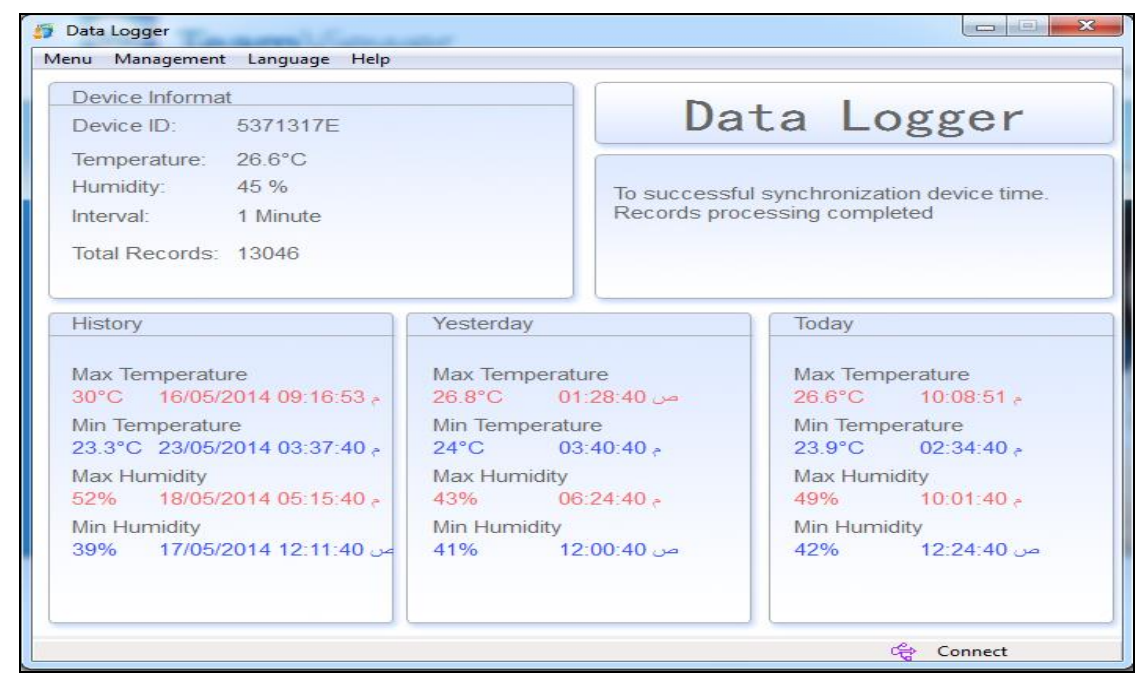

Fig. 2: Main intro page.

Fig. 2 shows the Main page for the program, which it display a summary of the data that recorded within the device. It contains the device information such as device ID, current temperature, humidity and the rate of change in minutes. In addition to the number of data, that recorded in the memory of the device. It also shows the last summary data such as high temperature and humidity were recorded in addition to the date on which it was recorded, as well as lower temperature and humidity. The second section of the Main page displayed the previous day and the current day. User can recognized that the program has been successfully linked with the device through the emergence of communication signal in a corner Program. This page contain also a taskbar to move around between pages programs.

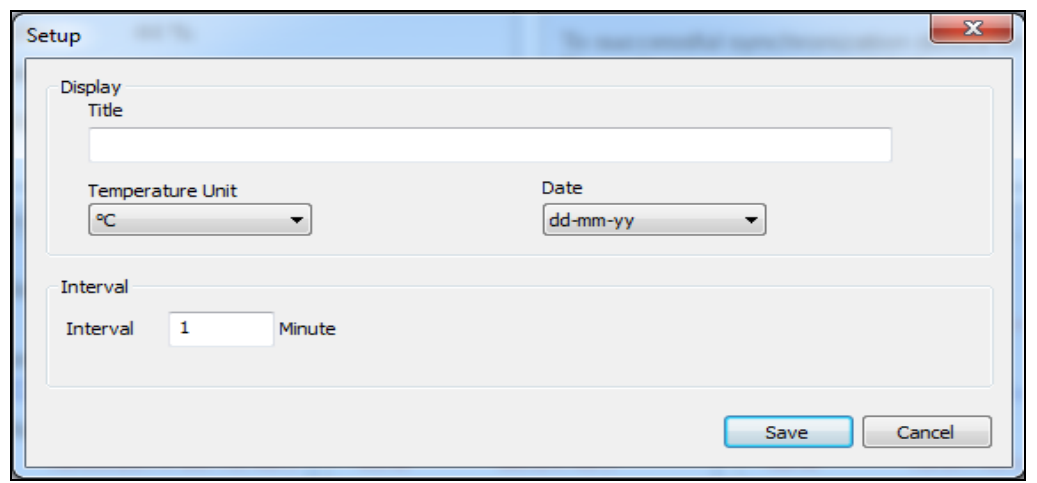

Fig. 3: Setup program and device.

Fig. 3, show the setup page, which is viewed by pressing the Menu button and then press Setup. User can control the temperature Unit, Date display system, in addition to the coefficient of interval time in the minutes. 


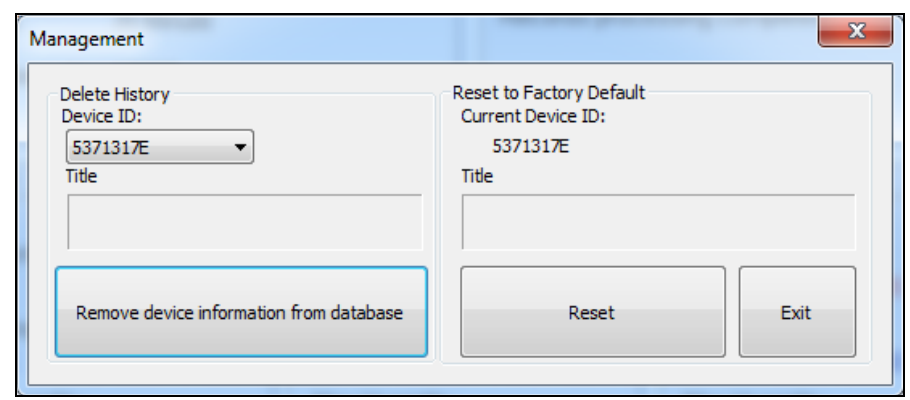

Fig. 4: Management of the device.

Fig. 4 shows the Management page. The user can view this page by clicking on the Management button located at the top of the taskbar of the main page of the program. This page provides the user to erase existing data that is stored in the memory the device reader. It stores the data of temperature and humidity with the device ID. The user can erase the records by clicking the left button appears on the screen "delete the device information from the database". In addition, it provides the user to restore the factory settings or the default settings of the program and the device.

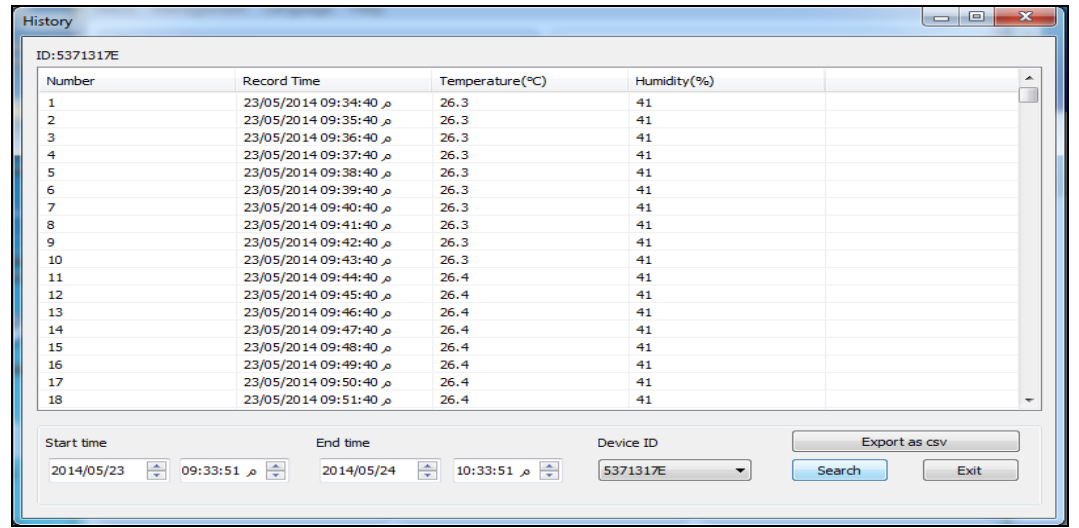

Fig. 5: Data history.

To view this screen (Fig. 5), the user should press the setup button and then history. This page shows a review of the data that is stored in the device memory, and selecting the start date and time, end date and time, then click on the Search button. In case date selection far can program takes the time to show results, all results that show us on the screen can be exported as an Excel file format.

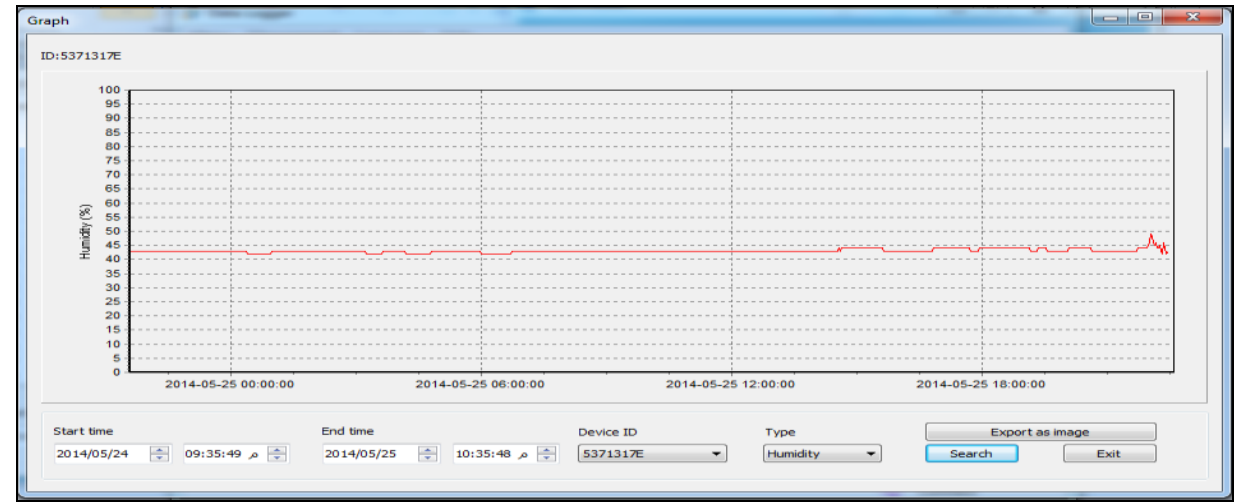

Fig. 6: Graph of the data.

In Fig. 6 can be shown by pressing the Setup button and then Graph. This page subjected the recorded data in the device memory in the form of drawing Graph by selecting the start date and time and end date and time, and then select the graphic. It is the temperature or the amount of moisture. Then click on the search button. Results can be exported in an image form. In this section's data is displayed in an Excel file form in order to facilitate the presentation of data and modify them. 


\section{Implementation}

While using the data logger device, it must set a date and time to be reading accurately and that may require sometimes plugging the device into the computer using the USB port. After making sure, that the device is working properly and is now equipped with senses that the temperature begins to take data. Senses temperature and moisture on an ongoing basis and because space high data memory where the intervention of this process infinitely, continuous and it is through the device calculating the amount of time that has passed for another read operation.

As shown in Fig. 7, it is re-reading every minute where the search continues in a minute if exceeded minute it records the data, and then goes back to search again. Then it stores the data in memory if the memory is full, the device is unable to re-reading so it separated from the sensor reading in and out of his throat continuous reading. Or it stopped in the event of an interrupt in the sequence of event data flow of the device through some roads. Such as the memory is full or the end of the battery or detach the device, which is based on the region where the reading.

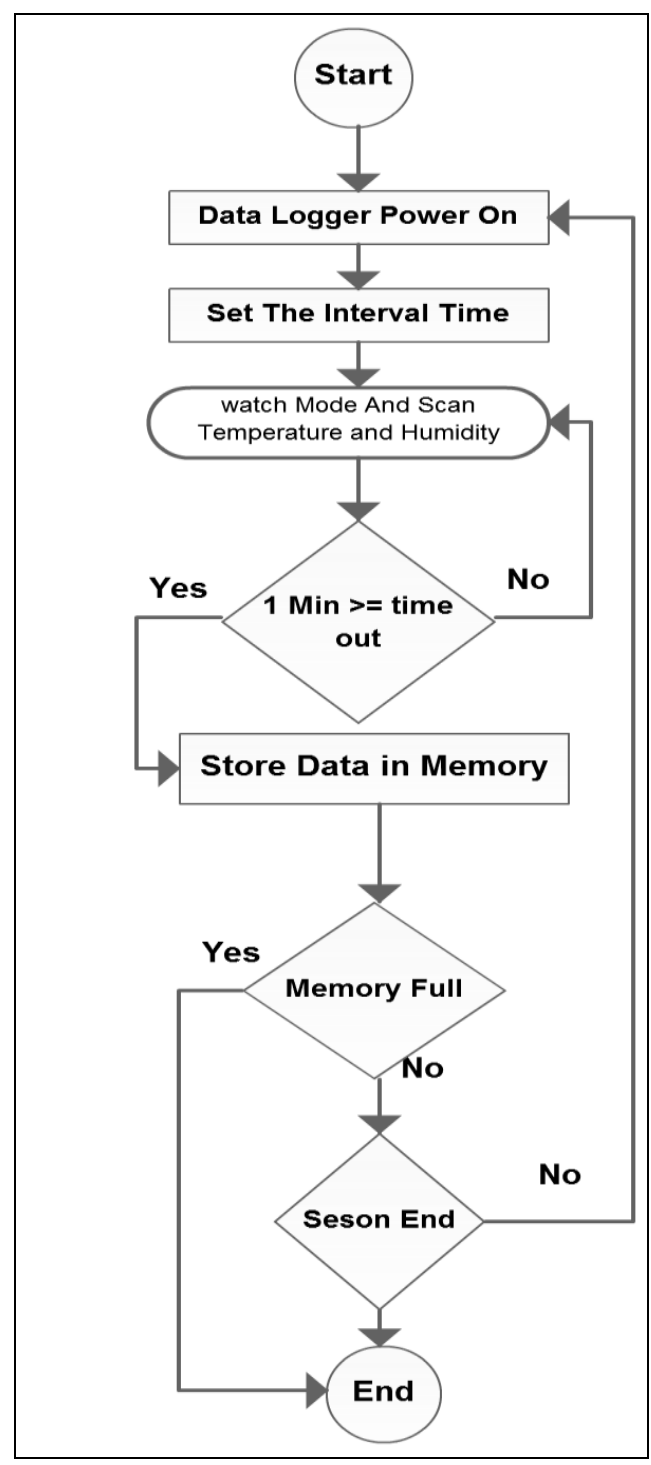

Fig. 7: Flow chart of data logger working.

\section{Results and Discussion}

In the measurement process, the program compares the accurate information from the device to know the amount or a variable amount or determine the status in any standard process. Fig. 8 shows the temperature and humidity results. 


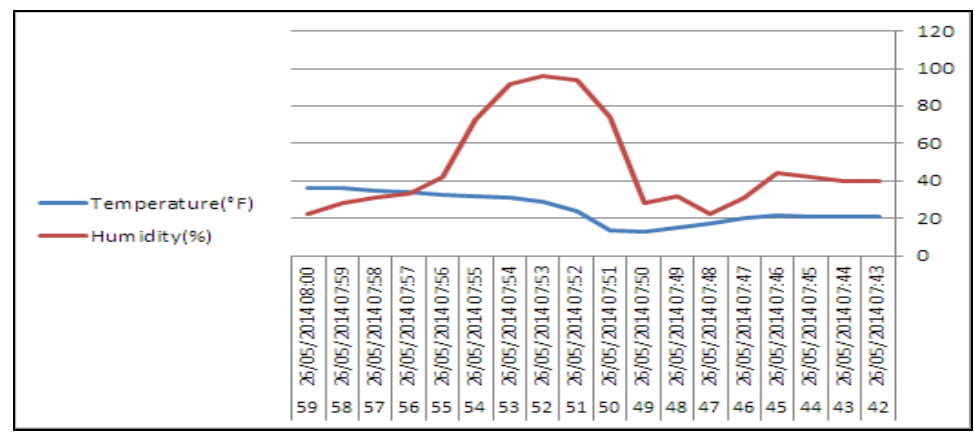

Fig. 8: Temperature and humidity results.

The change in temperature and humidity can be seen clearly in the experience, and that leading up to know the amount of change in temperature and humidity, as shown in Figure 8, which shows the chart for temperature and humidity result, where $\mathrm{X}$-Axis represents the time and the average change of time in minutes. And Y-Axis represents temperature and humidity with some Lama. Converting the energy to run the machine is not usually done without affecting the state of the thing. It cannot measure the degree of room temperature without putting the data recorder. Any process of measuring energy has been converted from one form to another may affect device on the quantity measured thus; the measurement must be pursuant careful precise aims to get information about something without affecting it. The numbers alone do not have a mean only if we have identified the units expressed. It is not enough to say that the temperature of the meat truck five, but we must say five degrees Celsius. Thus, we must mention the numbers and units of global or global derivatives units. The best temperatures for each food is shown in the following Table 1:

Table 1: Best Temperatures for Keeping Food Table

\begin{tabular}{|c|c|}
\hline Food stuffs chilled & Temperatures \\
\hline $\begin{array}{l}\text { Fresh sea products, especially fish, } \\
\text { crustaceans } \\
\text { Meat and meat chopped massacre in unit } \\
\text { sale to the consumer } \\
\text { Pre- cooked meals } \\
\text { Cold meals prepared on the same day, } \\
\text { sandwiches and gravy Summary } \\
\text { Modern -made } \\
\text { Refreshments, generous } \\
\text { Refreshments and fresh pastries } \\
\text { Poultry, rabbits, meat hunting } \\
\text { Products containing eggs } \\
\text { Eggs chilled } \\
\text { Raw milk, pasteurized } \\
\text { Products of fresh milk are pasteurize } \\
\text { fermented milk and cream, sweetener } \\
\text { Butter } \\
\text { Fresh cream, fresh cheese } \\
\text { Cheese with a soft dough, cheese, dough is } \\
\text { blended with parsley } \\
\text { Other cheese } \\
\text { Meat with bone and machining } \\
\text { Milk -oriented industry }\end{array}$ & $\begin{array}{c}+2^{\circ} \mathrm{C} \\
+3{ }^{\circ} \mathrm{C} \\
+3{ }^{\circ} \mathrm{C} \\
+3{ }^{\circ} \mathrm{C} \\
\\
+3{ }^{\circ} \mathrm{C} \\
+3{ }^{\circ} \mathrm{C} \\
+4{ }^{\circ} \mathrm{C} \\
+4{ }^{\circ} \mathrm{C} \\
+6{ }^{\circ} \mathrm{C} \\
+6{ }^{\circ} \mathrm{C} \\
+6{ }^{\circ} \mathrm{C} \\
\\
+10 \\
+15^{\circ} \mathrm{C} \\
+7^{\circ} \mathrm{C} \\
+8{ }^{\circ} \mathrm{C} \\
+10^{\circ} \mathrm{C} \\
+15^{\circ} \mathrm{C} \\
+15^{\circ} \mathrm{C}\end{array}$ \\
\hline
\end{tabular}

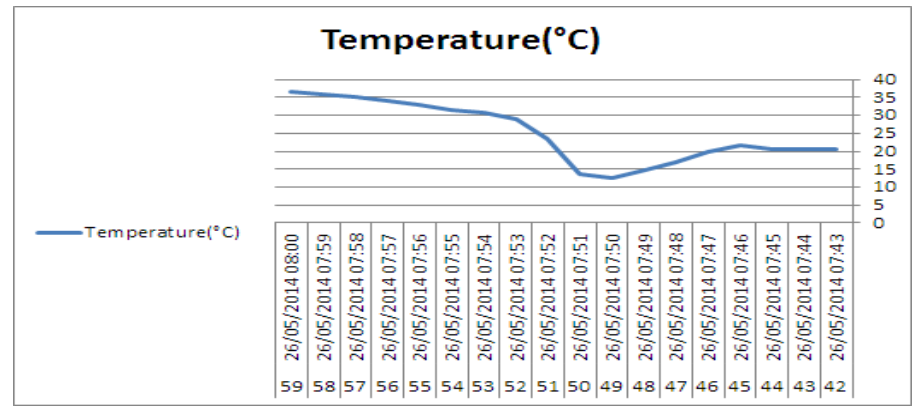

Fig. 9: Temperature results. 


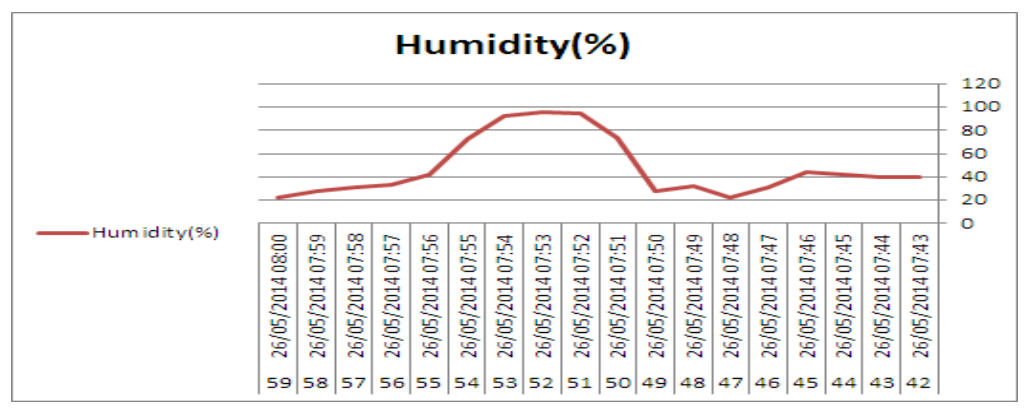

Fig. 10: Humidity results.

Fig. 9 clarified only the change of temperatures in order to understand the information and data correctly, where X-Axis represents the time and the average change of time in minutes. Moreover, Y-Axis represents degrees temperature. Nevertheless, Fig. 10, shows only the rate of change for the humidity in order to clarify the information and data correctly, where X-Axis represents the time rate of change and the time in minutes. Moreover, Y-Axis represents the rate of humidity and moisture measured using percentage.

\section{Conclusion and Future Work}

In this research, explained the problem arising from the changes in temperature and humidity, which affect the products and cause damage. In addition, it proposed to use a Data logger, which records the temperature and humidity and converted to graphical (whether temperature Celsius or Fahrenheit). The data logger device helped with treating the problem of the change in temperature and humidity, which causes damage to the product and cause damage to the health of the consumer, by giving accurate readings and temperature and humidity, in addition to ( the time and date ) since the device activated. Using the data logger device gives this result: the temperature in the freezer dropped to $12.7^{\circ} \mathrm{C}$, and the boiling water temperature $30^{\circ} \mathrm{C}$, the device in outside (regular) gives $36.5^{\circ} \mathrm{C}$ temperature.

We aspire to provide a data logger in places Electronics in Kuwait, and that is used in many institutions to maintain consumer protection, in the future we are working on the development of the machine and add other features to increase the efficiency of the device, even help in overcoming the problem of damage to products, consequent consumer protection.

\section{References}

[1] Burchfield, J. D., and GL Herries Davies. "Lord Kelvin and the Age of the Earth." (1994).

[2] Bishop, Michael L., Edward P. Fody, and Larry E. Schoeff, eds. Clinical Chemistry: Principles, Techniques, and Correlations. Lippincott Williams \& Wilkins, 2013.

[3] VanSomeren, Eus JW. "More than a marker: interaction between the circadian regulation of temperature and sleep, age-related changes, and treatment possibilities." Chronobiology international 17.3 (2000): 313-354.

[4] Normann, R. A., J. A. Henfling, and D. D. Blackwell. Development and field use of a memory-based pressure/temperature logging tool for the geothermal industry. No. DOE/EE--0106; CONF-9604116--30. Sandia National Laboratories (SNL-NM), Albuquerque, NM, 1996.

[5] van Marken Lichtenbelt, Wouter D., et al. "Evaluation of wireless determination of skin temperature using iButtons." Physiology \& Behavior88.4 (2006): 489-497.

[6] Kit L. Yam (October 2009). The Wiley Encyclopedia of Packaging Technology. New York: Wiley. p34-34.

[7] Porter, J., Arzberger, P., Braun, H. W., Bryant, P., Gage, S., Hansen, T., ... \& Michener, W. (2005). Wireless sensor networks for ecology. BioScience,55(7), 561-572.

[8] Hamdi Akram, in Minia university, Egypt "saving food with cooling and freezing" 2008 Available at: http://kenanaonline.com/users/AkrumHamdy/posts/74158.

[9] Laycock, James Andrew. "Food cooling compliance data logger." U.S. Patent No. 6,811,305. 2 Nov. 2004.

[10] Campbell Scientific, Inc (2014) Data loggers- As for functionality, all of our data loggers. 2014. 